\title{
Magdalena Miedzińska
}

Department of Clinical Oncology/Chemotherapy, Elbląska Hospital, MAGODENT Sp. z o.o., Warsaw, Poland

\section{EOTTD Meeting in Berlin, July 5-6, 2019}

\author{
Address for correspondence: \\ Lek. Magdalena Miedzińska \\ Oddział Onkologii Klinicznej/Chemioterapii \\ Szpital Elbląska, MAGODENT \\ ul. Szamocka 6, 01-748 Warszawa \\ e-mail: miedzinska.magda@gmail.com \\ Oncology in Clinical Practice \\ 2019, Vol. 15, No. 6, 339-341 \\ DOI: 10.5603/OCP.2019.0037 \\ Translation: dr n. med. Dariusz Stencel \\ Copyright ( 2019 Via Medica \\ ISSN 2450-1654
}

\begin{abstract}
At the beginning of July 2019 international conference of the European Organization for the Treatment of Trophoblastic Disease (EOTTD) took place in the one of the largest university hospitals in Europe, the Charite hospital in Berlin. The main goal of this year's EOTTD conference was to emphasize the role of instant and correct clinical, biochemical, pathomorphological, immunohistochemical and genetic diagnosis, conducting intensive, compliant with recommendations treatment and the need to assess the quality of life of patients. National registers, the set-up of reference centers and their cooperation should serve to achieve these goals. Key words: gestational trophoblastic disease, gestational trophoblastic neoplasm
\end{abstract}

Oncol Clin Pract 2019; 15, 6: 339-341
On July 5-6, 2019 another international EOTTD (The European Organization for Treatment of Trophoblastic Disease) conference took place. The meeting place was one of the largest university hospitals in $\mathrm{Eu}-$ rope, i.e. the Charité hospital in Berlin, and its host was Jalid Sehouli - professor of gynecology and oncology. As always, the meeting was chaired by prof. Michael Seckl from London. In total, 52 physicians and scientists, including four from Poland, took part in the conference. The only report from Poland was the speech of Dr. Grzegorz Szewczyk on the second day of the conference in the session: "New GTD center updates what is working and what needs fixing?".

The first day was dedicated to lecture sessions, and the second was the day of workshop meetings. The activity of the EOTTD group began in 2009. Its main goal was to bring together specialists dealing with Gestational Trophoblastic Disease (GTD) and Gestational Trophoblastic Neoplasm (GTN) and create current recommendations [1]. The disease mainly affects young women and despite common advanced clinical stage it should always be treated as potentially curable. However, despite good prognosis, it is estimated that every year around 2,100 patients diagnosed with GTN die due to inadequate medical care, which is mainly due to ignorance of biology and the course of the disease, as well as a limited group of specialists involved in its diagnosis and treatment.
During the lectures, the current recommendations and treatment options for GTN were presented, with well-established cytostatics and their combinations depending on the risk score [2]. In the low-risk group (0-6 points), monotherapy is recommended: methotrexate or dactinomycin. In the high-risk group (from 7 points), multi-drug chemotherapy with the EMA-CO regimen (etoposide, methotrexate, dactinomycin - cyclophosphamide, vincristine) with possible 2-3 induction EP cycles (etoposide, cisplatin) or EP-EMA regimen (etoposide, cisplatin - etoposide, methotrexate, dactinomycin). Particular attention should be paid to patients from the so-called "ultra-high risk" group (above 12 points), where the tumor doubling time is only a few days and it is necessary to start immediate, intensive treatment. The option available and used in special cases is high-dose chemotherapy with peripheral blood stem cell transplantation, which, despite its effectiveness, requires extreme caution due to possible complications, including death [3].

In connection with biological premises [4] and initial encouraging results of immunotherapy with use of immune response checkpoints inhibitors, most commonly pembrolizumab [5], the French center in 2017 began a multi-center phase II clinical study TROPHIMMUN, aiming to assess the efficacy and toxicity of avelumab in the treatment of chemotherapy-resistant GTN patients. Avelumab is anti-PD-L1 (programmed death 
ligand 1) monoclonal antibody and was administered at a dose of $10 \mathrm{mg} / \mathrm{kg}$ every 2 weeks until normalization of hCG concentration, followed by an additional three consolidation cycles. Preliminary results of the study will be presented during the ISSTD (The International Society for the Study of Trophoblastic Diseases) congress in Toronto in October this year.

The next most widely discussed issues were the rarest GTN subtypes, i.e. Placental Site Trophoblastic Tumor (PSTT) and Epithelioid Trophoblastic Tumor (ETT). A characteristic feature of these diseases, which significantly impedes and delays proper diagnosis, is usually a long time interval from the preceding pregnancy, calculated in months or even years. A detailed algorithm is presented depending on the stage of cancer and the main prognostic factor, which is the time since the previous pregnancy (the cut-off value was set at 48 months), as well as the desire to preserve fertility. Because of the usually high chemoresistance, surgery is the preferred treatment option, and if chemotherapy is needed, the EP/EMA or TP/TE multi-drug regimens (paclitaxel, cisplatinum/paclitaxel, etoposide) are used [6, 7]. It was recommended to report PSTT and ETT cases to the international register, which has already collected data on over 300 patients from 13 countries (http:// stdc.group.shef.ac.uk/psttuhr).

One of the sessions was devoted to patients' quality of life and widely understood psychosocial issues. Very interesting lectures was on fertility after GTN and cognitive impairment after chemotherapy. However, the presentations prepared by two patients after GTN treatment met with the greatest interest. They made us - doctors aware how the diagnostic process and finally the diagnosis and subsequent treatment affect not only the patient's health, but also her entire functioning in the family and society as well as her relatives. On the other hand, it was very encouraging to meet directly healthy women with GTN diagnosed in the past and after undergoing intensive, multi-stage and long-term treatment. One patient belonged to the high-risk group and the other to the group of "ultra-high risk" with brain metastases. Making possible something which seems to be impossible, e.g. to achieve complete recovery in patients with very advanced disease and numerous metastatic lesions adds motivation to action and to continue treatment despite subsequent progression and treatment toxicity. It should be emphasized, that GTN at every stage of treatment should always be treated as potentially curable.

The next session was the reports of representatives of the newly established national trophoblast disease treatment centers. The Polish center, as last year, was presented by doctor Grzegorz Szewczyk from the
Institute of Mother and Child in Warsaw, where, with the consent of the National Oncological Gynecology Consultant and with the support of the Polish Society of Oncological Gynecology, in 2017 the Trophoblast Disease Treatment Center was created, to which more and more women are reporting, usually after searching the center on the internet. Based on epidemiological data, it is estimated that in Poland there are about 600 cases of GTD every year and 60 to 80 cases of GTN. This small amount results in a large dispersion of patients and the resulting small practical experience of individual doctors. Grzegorz Szewczyk, in addition to presenting statistical data and development plans for the Center, has recently presented a registry regarding patients with GTD, thanks to which it will be possible in the future to assess both the morbidity and the results of GTN treatment in our country more precisely (www. trofoblast.imid.med.pl). Apart from Poland, speakers from Ireland, Serbia, Sweden and Ukraine presented their centers.

Traditionally, on the second day, workshops took place in subgroups: clinicians and far fewer pathomorphologists, biochemists and nurses. In the group of clinicians I participated in, apart from organizational matters, the focus was on agreeing treatment recommendations for patients with disease progression or relapse after treatment with EMA-CO multi-drug chemotherapy (postulated II line scheme is TP/TE, and III line treatment is pembrolizumab, which would replace high-dose chemotherapy with peripheral blood stem cell transplantation) and an algorithm for the diagnosis of atypical placental site nodule (APSN), which were previously considered benign, and as it turns out can be associated with the development of PSTT in as much as $10-15 \%$ and ETT. In each subgroup, the role of integrating the community of people dealing with patients diagnosed with GTD was highlighted in order to exchange experiences and create research opportunities. The final effect of the workshop are to be recommendations that, after agreeing with ESGO (The European Society of Gynecological Oncology) and IGCS (The International Gynecologic Cancer Society), are to be published at the end of this year and then updated every three years.

The main postulate of this year's EOTTD conference was to emphasize the role of rapid and correct clinical, biochemical, pathomorphological, immunohistochemical and genetic diagnosis, intensive treatment in accordance with the recommendations and the need to assess the quality of life of patients. National registers, creation of reference centers and their cooperation should serve to achieve the above objectives.

Next year's EOTTD conference will take place in Porto, the next in 2021 is planned in Warsaw. 


\section{References}

1. Bolze PA, Attia J, Massardier J, et al. EOTTD group. Formalised consensus of the European Organisation for Treatment of Trophoblastic Diseases on management of gestational trophoblastic diseases. Eur J Cancer. 2015; 51(13): 1725-1731, doi: 10.1016/j.ejca.2015.05.026, indexed in Pubmed: 26092638.

2. FIGO Oncology Committee report: FIGO staging for gestational trophoblastic neoplasia 2000. Int J Gynecol Obstet. 2002; 77(3): 285-287, doi: 10.1016/s0020-7292(02)00063-2.

3. Frijstein MM, Lok CAR, Short D, et al. The results of treatment with high-dose chemotherapy and peripheral blood stem cell support for gestational trophoblastic neoplasia. Eur J Cancer. 2019; 109: 162-171, doi: 10.1016/j.ejca.2018.12.033, indexed in Pubmed: 30731277.

4. Bolze PA, Patrier S, Massardier J, et al. PD-L1 expression in premalignant and malignant trophoblasts from gestational trophoblastic diseas- es is ubiquitous and independent of clinical outcomes. Int J Gynecol Cancer. 2017; 27(3): 554-561, doi: 10.1097/IGC.0000000000000892. indexed in Pubmed: 28060141

5. Ghorani E, Kaur B, Fisher RA, et al. Pembrolizumab is effective for drug-resistant gestational trophoblastic neoplasia. Lancet. 2017 390(10110): 2343-2345, doi: 10.1016/S0140-6736(17)32894-5, indexed in Pubmed: 29185430.

6. Frijstein MM, Lok CAR, van Trommel NE, et al. Management and prognostic factors of epithelioid trophoblastic tumors: Results from the International Society for the Study of Trophoblastic Diseases database. Gynecol Oncol. 2019; 152(2): 361-367, doi: 10.1016/j. ygyno.2018.11.015, indexed in Pubmed: 30473257.

7. Gadducci A, Carinelli S, Guerrieri ME, et al. Placental site trophoblastic tumor and epithelioid trophoblastic tumor: Clinical and pathological features, prognostic variables and treatment strategy. Gynecol Oncol. 2019; 153(3): 684-693, doi: 10.1016/j.ygyno.2019.03.011, indexed in Pubmed: 31047719 . 